\title{
Numerical Simulation of the Vibration Behavior of Curved Carbon Nanotubes
}

\author{
Sadegh Imani Yengejeh, ${ }^{1}$ João M. P. Q. Delgado, ${ }^{2}$ \\ Antonio G. Barbosa de Lima, ${ }^{3}$ and Andreas Öchsner ${ }^{4,5}$ \\ ${ }^{1}$ Department of Solid Mechanics and Design, Faculty of Mechanical Engineering, Universiti Teknologi Malaysia (UTM), \\ 81310 Skudai, Johor, Malaysia \\ ${ }^{2}$ Laboratory of Building Physics (LFC), Civil Engineering Department, Faculty of Engineering, University of Porto, $4200-465$ Porto, \\ Portugal \\ ${ }^{3}$ Department of Mechanical Engineering, Federal University of Campina Grande, 58429-900 Campina Grande, PB, Brazil, Brazil \\ ${ }^{4}$ School of Engineering, Griffith University, Gold Coast Campus, Southport, QLS 4222, Australia \\ ${ }^{5}$ School of Engineering, The University of Newcastle, Callaghan, NSW 2308, Australia
}

Correspondence should be addressed to Sadegh Imani Yengejeh; imani.sd@gmail.com

Received 20 February 2014; Accepted 22 April 2014; Published 29 May 2014

Academic Editor: Bin Li

Copyright (C) 2014 Sadegh Imani Yengejeh et al. This is an open access article distributed under the Creative Commons Attribution License, which permits unrestricted use, distribution, and reproduction in any medium, provided the original work is properly cited.

\begin{abstract}
Several zigzag and armchair single-walled carbon nanotubes (CNTs) were modeled by a commercial finite element package and their vibrational behavior was studied. Numerous computational tests with different boundary conditions and different bending angles were performed. Both computational and analytical results were compared. It was shown that the computational results are in good agreement with the analytical calculations in the case of straight tubes. In addition, it was concluded that the natural frequency of straight armchair and zigzag CNTs increases by increasing the chiral number of both armchair and zigzag CNTs. It was also revealed that the natural frequency of CNTs with higher chirality decreases by introducing bending angles. Nevertheless, the influence of increasing bending angle on the natural frequency of armchair and zigzag CNTs with lower chiral number is almost negligible.
\end{abstract}

\section{Introduction}

Since the discovery of carbon nanotubes (CNTs) by Iijima in 1991 [1], these nanostructures have been the focus of many investigations. CNTs are important to industrial applications because of their outstanding mechanical and physical properties such as strength and lightness. The investigations on CNTs can be divided into two groups of experimental and computational approaches. Molecular dynamics (MD) and continuum mechanics approach such as the finite element method (FEM) have been the most popular approaches to study the behavior, for example, the vibrational behavior, of these nanoparticles [2]. In the following, the results of several studies on the evaluation of CNTs vibrational properties are presented.
In 2009, Georgantzinos et al. [3] proposed a linear springbased element formulation for the computation of vibrational characteristics of single-walled carbon nanotubes (SWCNTs). They developed three-dimensional nanoscale elements and corresponding elemental equations for the numerical treatment of the dynamic behavior, that is, appropriate stiffness and mass characteristics, of SWCNTs. They also assembled the elemental equations and constructed the dynamic equilibrium equation, applying the atomic microstructure of CNTs. The developed elements in their investigations simulated the relative translations and relation between atoms. Consequently, they could apply the molecular mechanics theory directly due to the modeling of atomic bonds, applying physical variables such as bond stretching. Based on their results, new natural frequencies and mode shapes for 
numerous CNTs under different boundary conditions were found. They also showed that their results indicated very good agreement compared to numerical predictions from the literature. In 2010, Xia and Wang [4] investigated the vibration characteristics of fluid-conveying carbon nanotubes with a curved longitudinal structure. They could obtain the natural frequencies of curved carbon nanotubes conveying fluid and compared them with those of straight CNTs. Their findings revealed that CNTs with circular curved longitudinal shape are unconditionally stable even for a system with sufficiently high flow velocity. In 2011, Arghavan and Singh [5] presented a detailed numerical study on the free and forced vibration of straight SWCNTs. They analyzed both armchair and zigzag CNTs with clamped-clamped and clamped-free boundary conditions in order to find their natural frequencies and corresponding mode shapes. Their results indicated the appearance of these modes of vibration in the eigenvalues and eigenvectors without any distinction. They finally concluded that in the case of zigzag CNTs, the axial modes appeared to be decoupled whereas the armchair nanotubes show coupling between such modes. Although they thoroughly investigated the behavior of straight CNTs, the study on the behavior of curved nanotubes is only rarely addressed. Ghavamian and Öchsner [6] investigated the eigenvalues and eigenfrequencies of CNTs under the influence of defects in 2013. After simulating armchair and zigzag configurations of zigzag and armchair CNTs based on the FEM, they introduced three most likely defects to the models in order to represent defective forms of SWCNTs. These defects are carbon vacancy, Si-doping, and perturbation. Finally they examined and also compared the vibrational behavior of perfect and defective CNTs. According to their findings, SWCNTs have a natural frequency in the range of 18.69 to 24.01 GHz. In addition, it was pointed out that the existence of any type of defects leads to a lower value of natural frequency and vibrational stability. The aim of this study is to continue the previous investigations and to focus on the evaluation and comparison of vibrational behavior of curved CNTs with different bending angles. Since experimental observations (e.g., based on electron microscopes) show that CNTs are not usually straight, but rather have certain degree of curvature or waviness along the nanotubes length $[7,8]$, we focus in the following on the investigation of the effect of the curvature on the dynamic behavior. All our simulations were performed with the commercial finite element code MSC.MARC (MSC Software Corporation, Santa Ana, CA, USA).

\section{Methodology}

2.1. Geometric Definition. CNTs are assumed to be some kind of hollow cylinder shape configurations, as shown in Figure 1. These special nanostructures can be imagined by rolling a graphene sheet into a cylinder. They possess a length over $10 \mu \mathrm{m}$ and diameters ranging from 1 to $50 \mathrm{~nm}$. The geometry of a CNT is defined by the chiral vector $\vec{C}_{h}$ and the chiral angle $\theta$. The chiral vector is presented by two unit vectors $\vec{a}_{1}$ and $\vec{a}_{2}$ and two integers $m$ and $n$ as it is presented by the following equation [9]:

$$
\vec{C}_{h}=n \vec{a}_{1}+m \vec{a}_{2}
$$

The construction of CNTs is defined based on the chiral vector or angle by which the sheet is rolled into a cylinder, in three different configurations including chiral, zigzag, and armchair. In the case of $\left(\theta=0^{\circ}\right)$ or $(m=0)$ the zigzag CNT is constructed. An armchair CNT is obtained in terms of chiral vector $(m=n)$ or in terms of chiral angle $\left(\theta=30^{\circ}\right)$, and finally a chiral CNT is shaped if $0^{\circ}<\theta<30^{\circ}$ or $m \neq n \neq 0$ [9].

Based on the following equation, the diameter of the CNT can be calculated:

$$
d_{\mathrm{CNT}}=\frac{a_{0} \sqrt{m^{2}+m n+n^{2}}}{\pi},
$$

where $a_{0}=\sqrt{3} b$ and $b=0.142 \mathrm{~nm}$ is the length of the C-C bond [9].

The modeling method presented in this study follows the idea first suggested in [10] where the theory of classical structural mechanics was extended into the modeling of CNTs. In a CNT, carbon atoms are bonded together by covalent bonds which have their characteristic lengths and angles in a three-dimensional space. Afterwards, it was suggested that CNTs, when subjected to loading, act as spaceframe structures. Therefore, the bonds between carbon atoms are considered as connecting load-carrying generalized beam members, while the carbon atoms behave as joints of the members. This idea is schematically shown in Figure 1.

In this study, the configurations of both armchair and zigzag SWCNTs were simulated by the CoNTub software [11]. Defining the chirality and the length of the tubes, the spatial coordinates of the $\mathrm{C}$-atoms and the corresponding connectivities (i.e., the primary bonds between two nearestneighboring atoms) were calculated. Then the gathered data was transferred to a commercial finite element package, where $\mathrm{C}-\mathrm{C}$ bonds were modeled as circular beam elements [12]. Afterwards, the FE analyses were conducted and vibrational behavior of different armchair and zigzag CNTs was evaluated.

The natural frequency is the frequency of a vibrating system at which the system oscillates at greater amplitude due to existence of the resonance phenomenon. Geometry, mass, and applied boundary conditions are the factors which influence these quantities. The natural frequency is mainly evaluated to examine the vibrational behavior of structural members. The first natural frequency of an Euler-Bernoulli beam element under different boundary condition is defined by the following equation [13]:

$$
f=\left(\frac{A}{2 \pi}\right) \sqrt{\frac{E I}{\bar{m} L^{4}}},
$$

where $E, I, \bar{m}$, and $L$ are the Young's modulus, the second moment of area, the mass per unit length, and the length of the nanotube, respectively. The $A$ value for fixed-free, fixedfixed, and free-free boundary condition is 3.5156, 22.373, and 22.4 , respectively. 


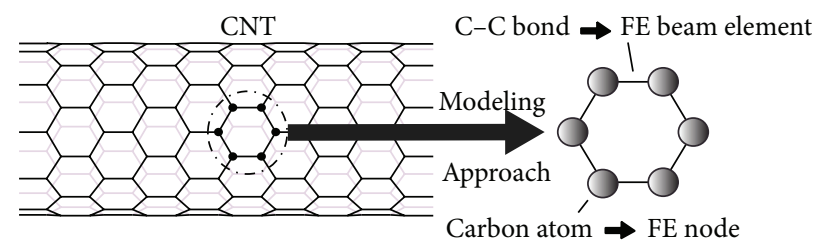

Figure 1: Side view of the $(11,11) \mathrm{CNT}$ as a space-frame structure.

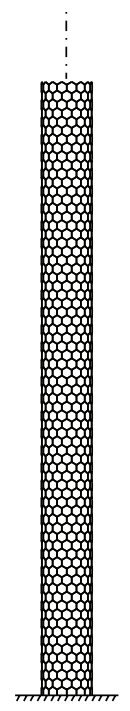

(a)

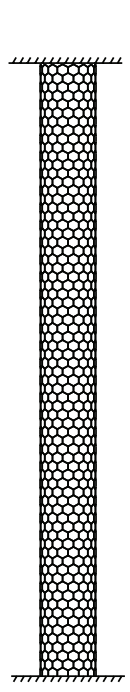

(b)

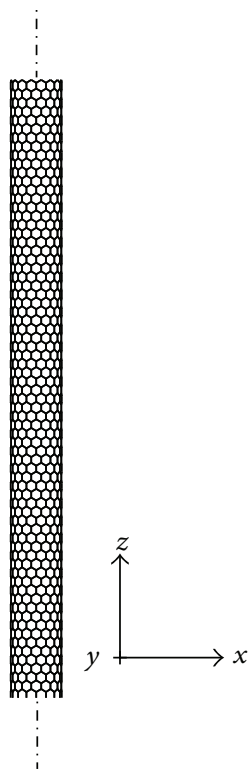

(c)

Figure 2: $(16,0)$ zigzag CNT with (a) fixed-free, (b) fixed-fixed, and (c) free-free boundary conditions.

2.2. Material Parameters and Boundary Conditions. In this study, the vibrational behavior of numerous types of armchair and zigzag SWCNTs under fixed-free, fixed-fixed, and freefree boundary conditions is investigated, in which one end is either free or fully fixed while the other end is fixed in the first two cases and for the third case both ends are completely free, as illustrated in Figure 2.

Figure 3 illustrates an armchair CNT under fixed-free boundary condition. The models of CNTs were simulated from straight CNT to curved CNT with $45^{\circ}$ bending angle, as shown in Figure 3.

For defining the properties of the equivalent beam elements for the CNT bonds, no such classical examinations or geometric derivations are available. As a result, the same values for the equivalent beam elements are assumed as in the approach proposed in $[10,15,16]$. These effective material and geometrical properties were found in the mentioned references based on a molecular mechanics method. Their motions are regulated by a force field, which is generated by electron-nucleus interactions and nucleus-nucleus interactions, and frequently expressed in the form of steric potential energy. This steric potential energy is in general the sum of contributions from bond stretch interaction, bond angle bending, dihedral angle torsion, and improper (out of plane) torsion as illustrated in Figure 4.
The introduced constants and the element properties of CNTs (armchair and zigzag) are listed in Table 1.

\section{Results and Discussion}

The vibrational behavior of different armchair and zigzag SWCNTs was investigated for different curvatures from $0^{\circ}$ to $45^{\circ}$ bending angle (fixed-free, fixed-fixed, and free-free boundary condition). After evaluating their natural frequencies from FEM, the results were compared to analytical calculations given by (3) in the case of straight configurations in order to have some kind of validation of the FE approach. Figure 5 illustrates the first mode of vibration of a (16-0) armchair CNT under different boundary conditions.

The relative difference between the analytical solution and FEM results of straight CNTs was defined by the following equation:

Relative difference in \%

$$
=\left|\frac{\text { FEM result }- \text { analytical solution }}{\text { analytical solution }}\right| \times 100 \text {. }
$$

This difference is listed in Table 2 for the case of the fixed-free boundary condition. Based on the results, it can be concluded that the computational solutions are in 


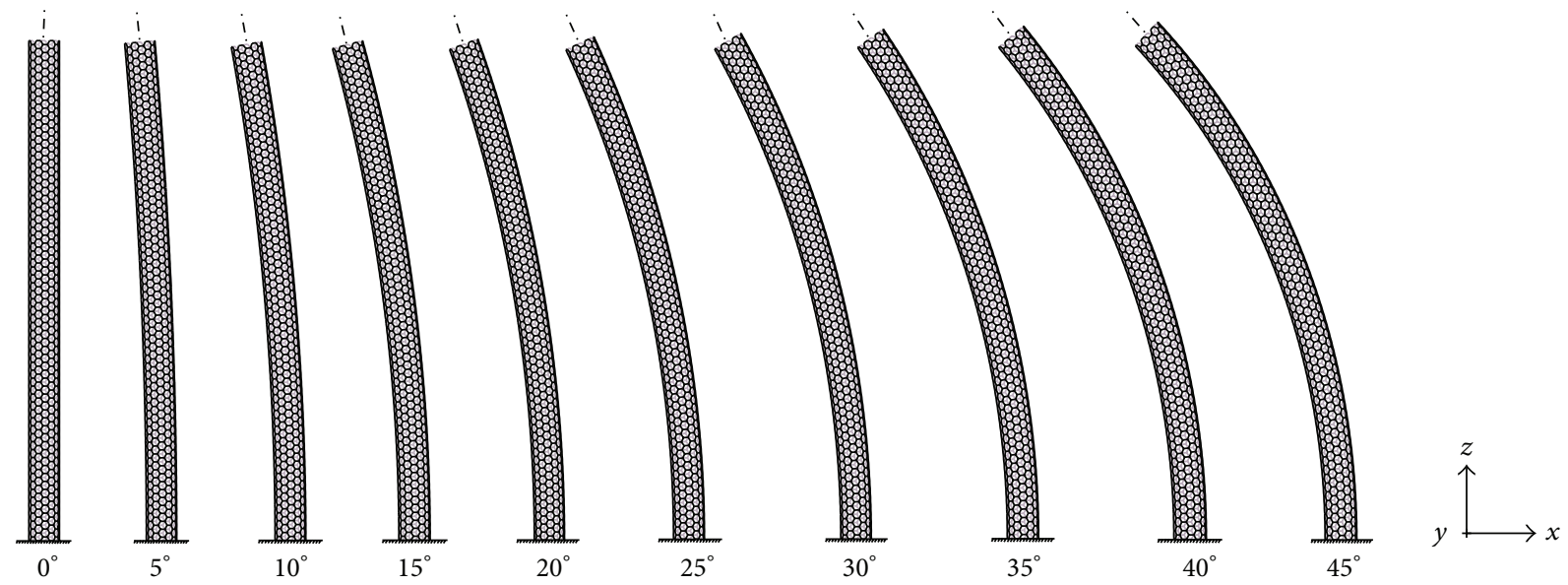

FIGURE 3: (7-7) armchair CNTs with fixed-free boundary condition and different forms from $0^{\circ}$ to $45^{\circ}$ bending angle.

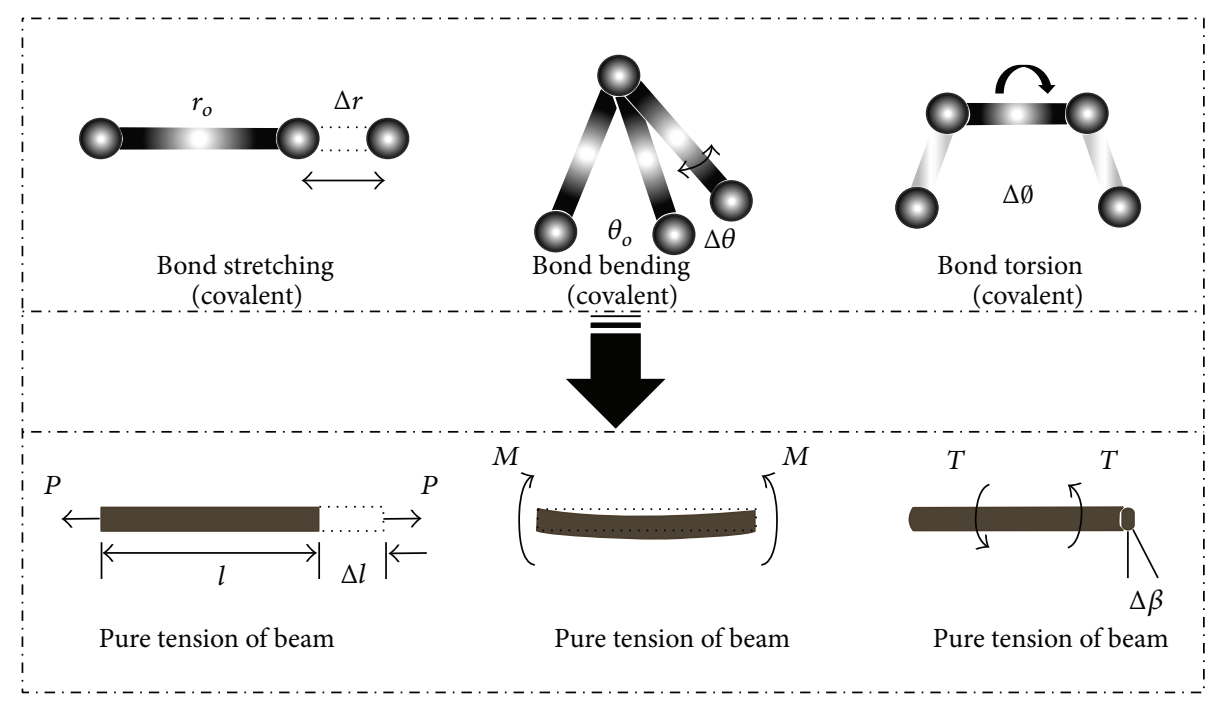

FIGURE 4: Equivalence of molecular mechanics and structural mechanics for covalent and noncovalent interactions between carbon atoms. Molecular mechanics model (up) and structural mechanics model (down).

TABLE 1: Material and geometric properties of C-C covalent bonds [14].

\begin{tabular}{lc}
\hline Corresponding force field constants & $k_{r}=651.97 \mathrm{nN} / \mathrm{nm}$ \\
$E=$ Young's modulus $=\frac{k_{r}{ }^{2} b}{4 \pi k_{\theta}}$ & $k_{\theta}=0.8758 \mathrm{nN} \mathrm{nm} / \mathrm{rad}^{2}$ \\
$G=$ shear modulus $=\frac{E}{2(v+1)}$ & $k_{\varphi}=0.2780 \mathrm{nN} \mathrm{nm} / \mathrm{rad}^{2}$ \\
$R_{b}=$ bond radius $=2 \sqrt{\frac{k_{\theta}}{k_{r}}}$ & $5.484 \times 10^{-6} \mathrm{~N} / \mathrm{nm}^{2}$ \\
$I_{x x}=I_{y y}=$ second moments of area $=\frac{\pi R_{b}{ }^{4}}{4}$ & $2.159 \times 10^{-6} \mathrm{~N} / \mathrm{nm}^{2}$ \\
\hline
\end{tabular}




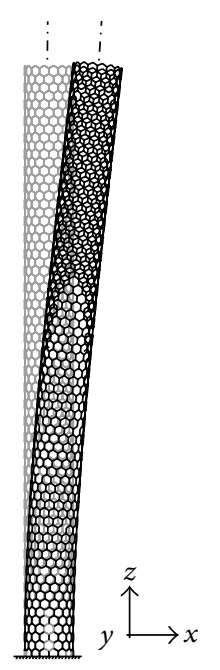

(a)

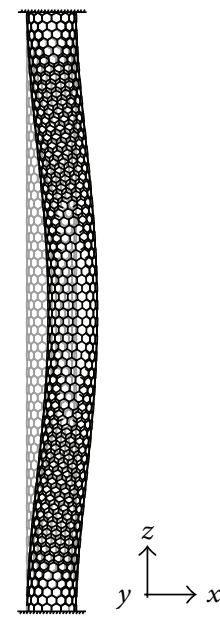

(b)

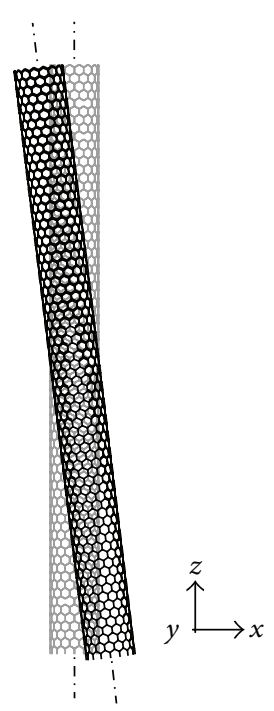

(c)

FIGURE 5: First eigenmode of a (16-0) straight CNT under (a) fixed-free, (b) fixed-fixed, and (c) free-free boundary conditions.

TABLE 2: Characteristics of simulated straight CNTs.

\begin{tabular}{lccccccc}
\hline CNT type & Chirality $(n, m)$ & Length $(\mathrm{nm})$ & Diameter $(\mathrm{nm})$ & Young's modulus (TPa) & $\begin{array}{c}\text { Natural frequency }(\mathrm{GHz}) \\
\text { Analytical } \\
\text { solution }\end{array}$ & $\begin{array}{c}\text { Relative } \\
\text { FEM result }\end{array}$ \\
& & & & & 1.039 & 10.01 & 8.47 \\
difference in (\%)
\end{tabular}

good agreement with the analytical calculations for the cantilevered boundary condition.

Each mode shape shows a significant natural frequency and eigenmode based on the boundary condition of the CNTs. For instance, the first four eigenmodes of a $(7,7)$ armchair CNT are shown in Figure 6.

Having a closer look on the results, it was revealed that the natural frequency of straight armchair and zigzag CNTs increases by increasing the chiral number of both armchair and zigzag CNTs, as illustrated in Figure 7.
It is also obvious that the natural frequency of both armchair and zigzag CNTs varied in a high value range. Comparison with other computational approaches based on a linear spring-based element formulation for straight CNTs [3] indicates a similar range of the frequencies.

It is clear that the existence of any geometrical imperfection, bending angle in particular, in the structure of armchair and zigzag straight CNTs results in a reduction in the natural frequency by increasing the bending angle, as illustrated in Figure 8. The natural frequency decreases for all cases of 


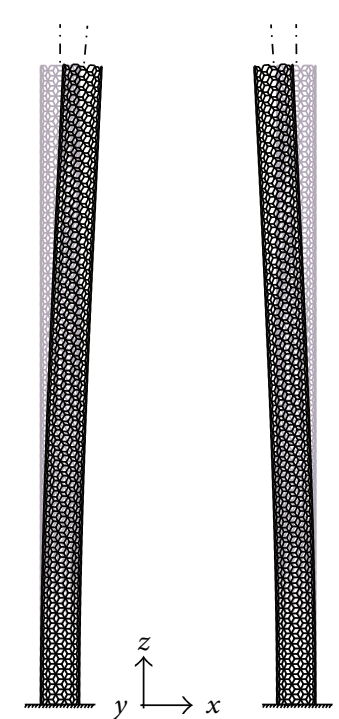

(a)

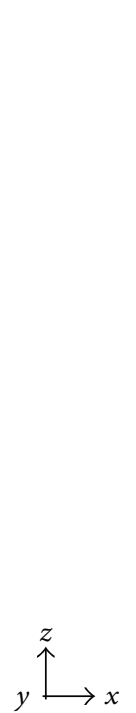

(b)

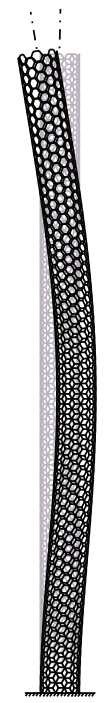

$y$

(c)

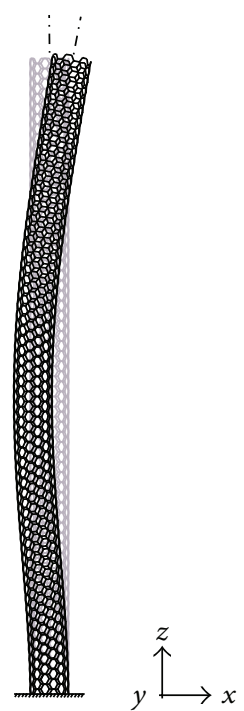

(d)

FIGURE 6: First four eigenmodes of a (7-7) straight CNT.

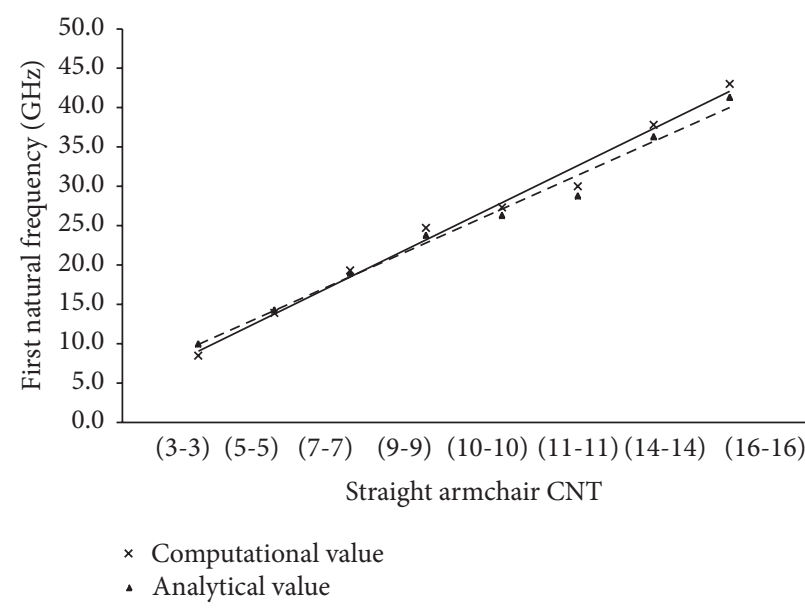

(a)

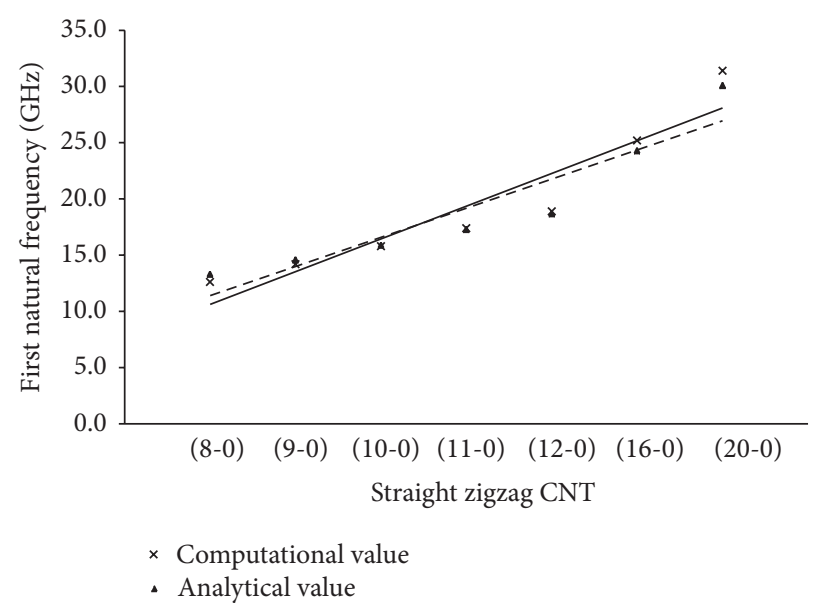

(b)

FIGURE 7: (a) Change in natural frequency for straight armchair CNTs. (b) Change in natural frequency for straight zigzag CNTs. The markers represent the results of the computation; the straight lines are linear regressions.

boundary conditions. However, it should be noted that this trend is more obvious for the CNTs with higher chirality, as the decrease of natural frequency for both armchair and zigzag CNTs with lower chirality is almost negligible. It is also indicated that the gradual decrease for the fixed-fixed and free-free case of boundary condition is more significant compared to the fixed-free case at higher curvatures. In addition, this trend is more obvious for the armchair CNTs.

Since higher natural frequencies may be important in some applications such as mass sensing at nanoscale [17], we exemplarily evaluated the second and third natural frequency and the corresponding mode shapes for the case of three armchair nanotubes, see Figure 9.

Comparison with Figure 8(a) shows that the second natural frequency is practically the same as the first one.
However, the third natural frequency is approximately five times higher than the first natural frequency. Furthermore, the trend line for the higher frequencies is similar to the one from the first natural frequency. An example of the corresponding mode shapes is presented in Figure 10.

\section{Conclusions}

In this study, numerous CNTs (zigzag and armchair) were simulated by an FE approach and their vibrational behavior was examined through performing several computational tests with different boundary conditions and variable bending angles. Towards achieving the most accurate results the vibrational behaviors of these CNTs were evaluated analytically and computationally and were compared together. 


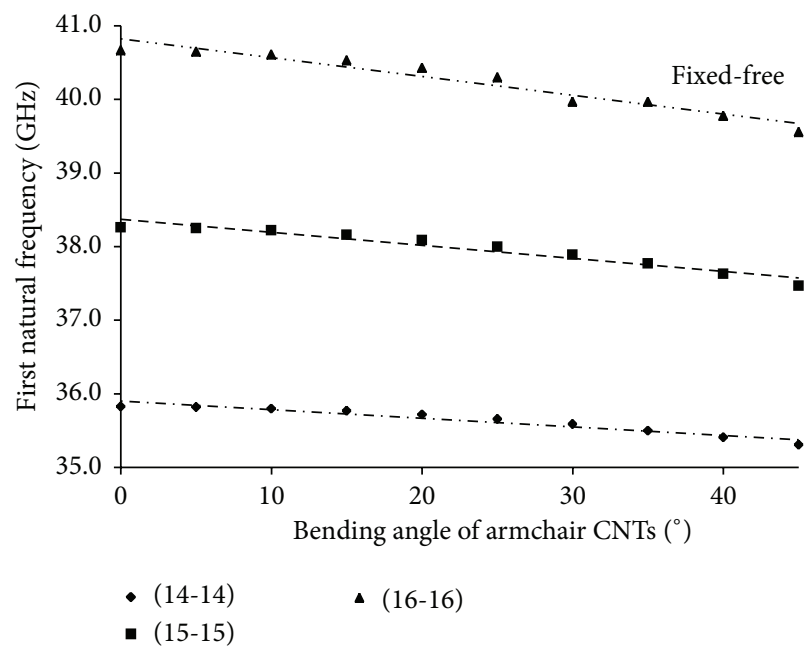

(a)

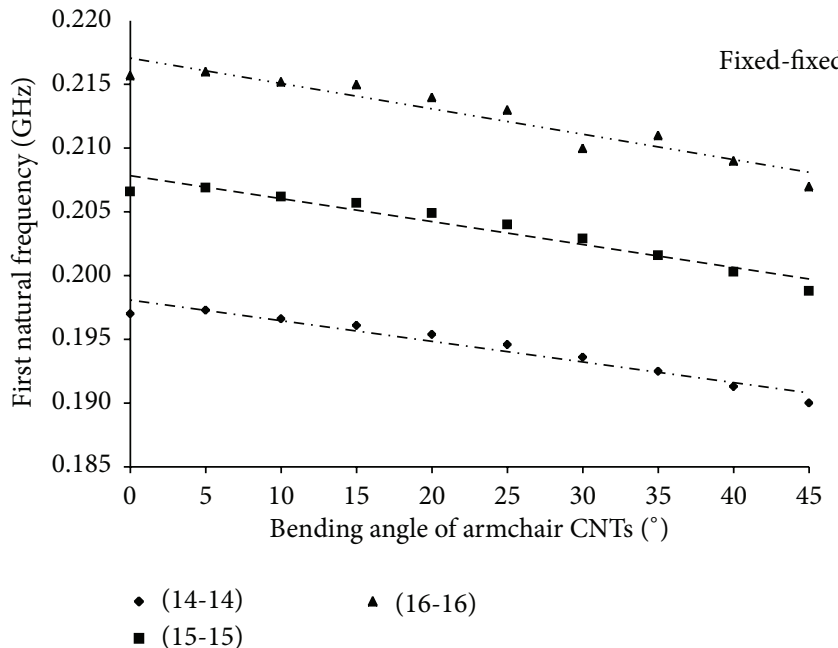

(c)

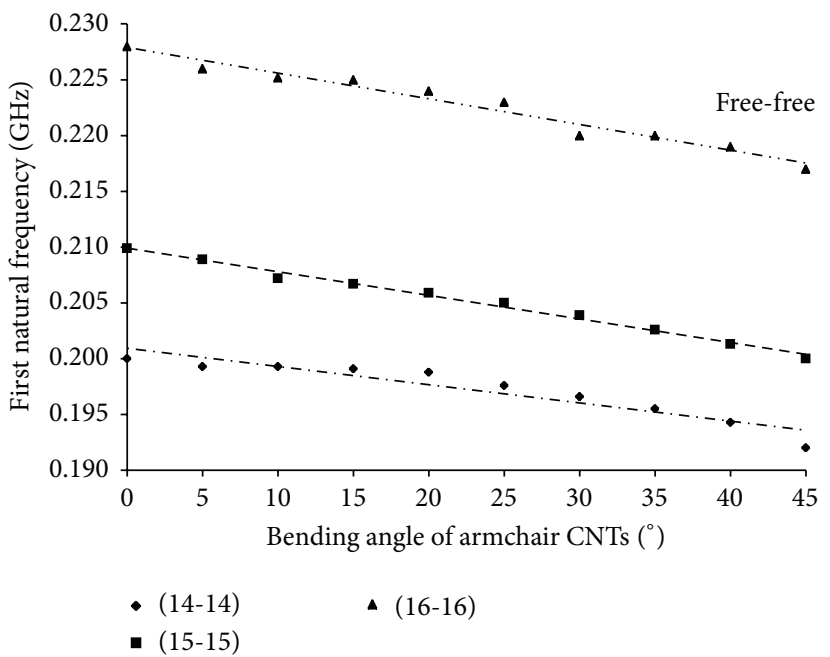

(e)

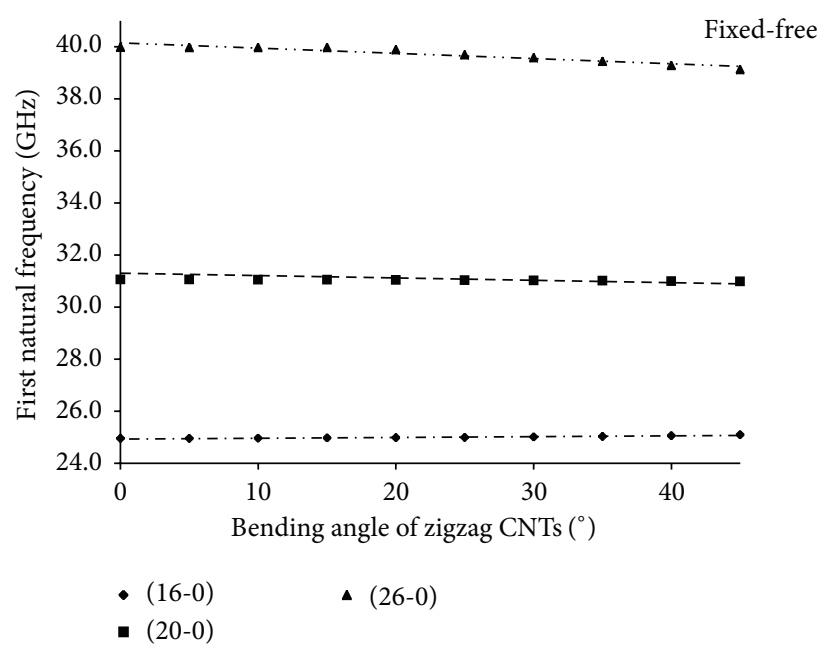

(b)

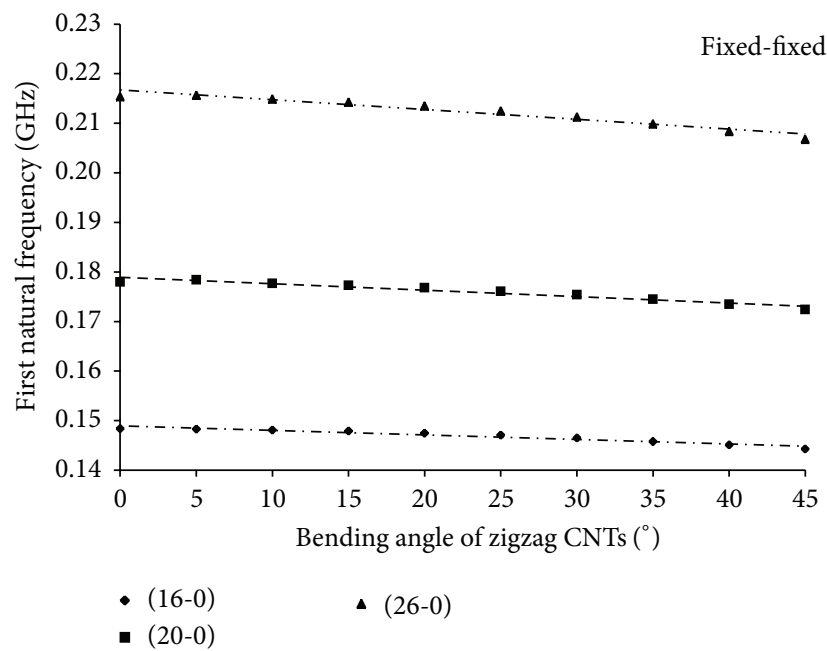

(d)

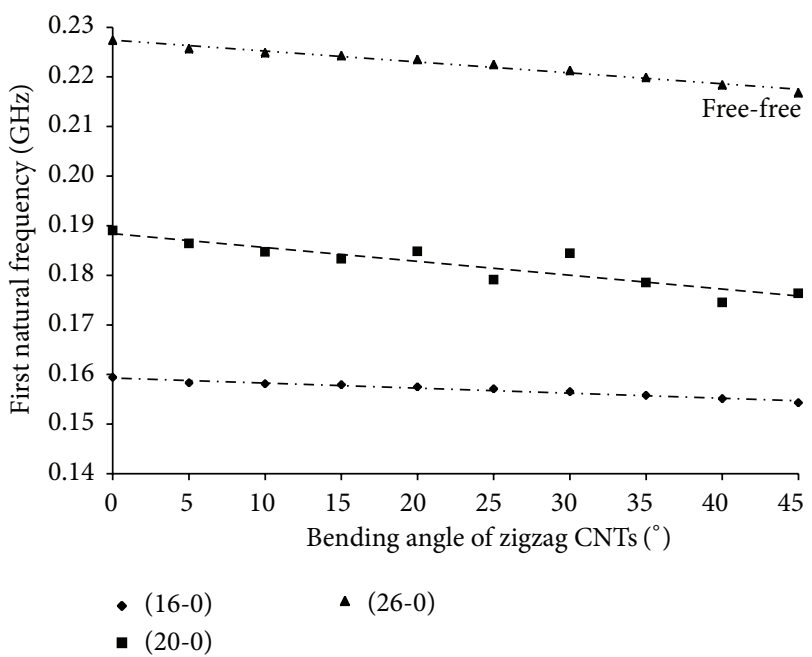

(f)

FIGURE 8: Change in natural frequency with (a) armchair and (b) zigzag CNTs for fixed-free, with (c) armchair and (d) zigzag CNTs for fixed-fixed and with (e) armchair and (f) zigzag for free-free boundary conditions. The markers represent the results of the computation; the straight lines are linear regressions. 


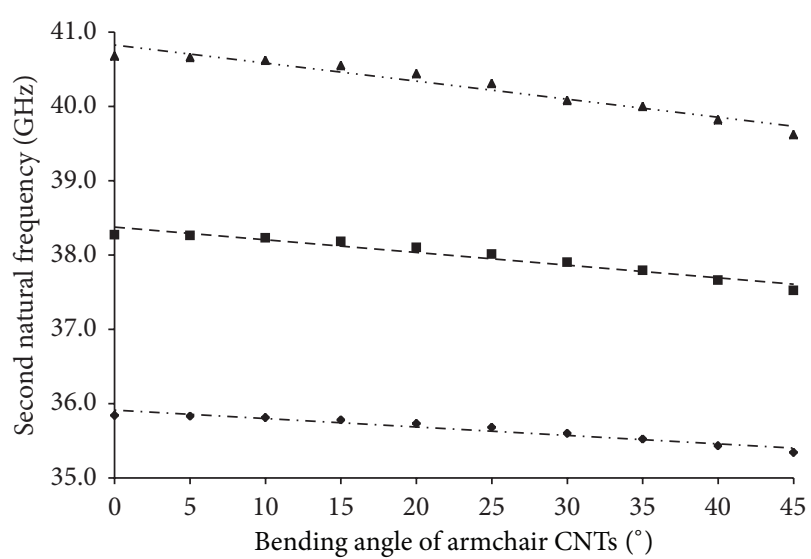

- (14-14)

- (15-15)

- (16-16)

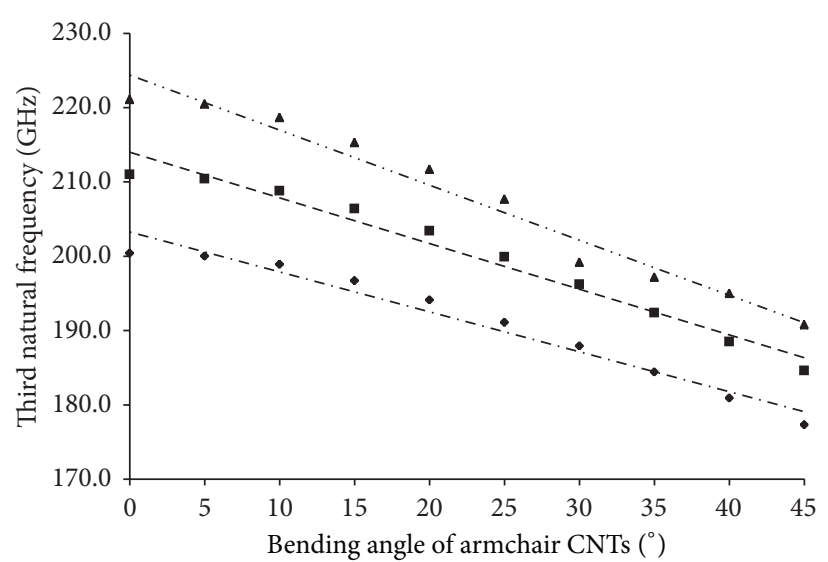

- (14-14)

- (15-15)

\ (16-16)

(a)

(b)

FIGURE 9: Change in higher natural frequency with armchair CNTs for fixed-free boundary condition: (a) second natural frequency and (b) third natural frequency. The markers represent the results of the computation; the straight lines are linear regressions.
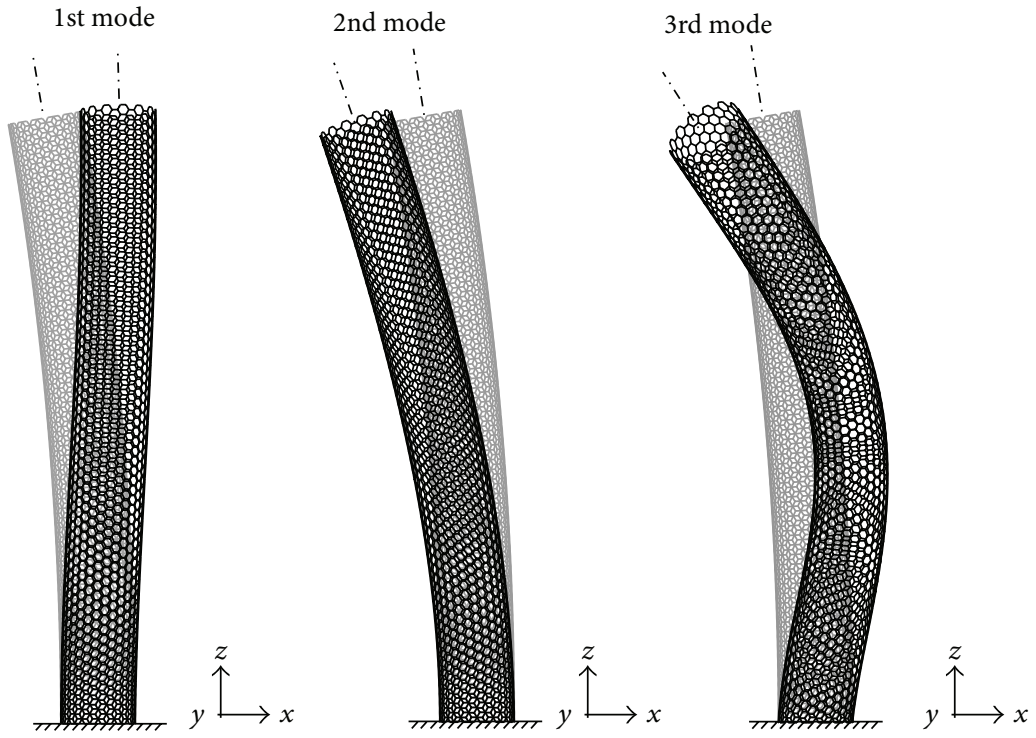

FIGURE 10: First three eigenmodes of a (14-14) CNT with $10^{\circ}$ bending angle.

It was concluded that the natural frequency of straight CNTs increases by increasing the chiral number of both armchair and zigzag CNTs. It was also revealed that the natural frequency of CNTs (zigzag and armchair) with higher chirality decreases by introducing bending angles for all cases of boundary conditions. However, the change of increasing the bending angle on the natural frequency of armchair and zigzag CNTs with lower number of chirality is almost negligible. The finding of this study may have useful effects on further investigations of CNTs.

\section{Conflict of Interests}

The authors declare that there is no conflict of interests regarding the publication of this paper.

\section{References}

[1] S. Iijima, "Helical microtubules of graphitic carbon," Nature, vol. 354, no. 6348, pp. 56-58, 1991. 
[2] S. Imani Yengejeh, M. Akbar Zadeh, and A. Öchsner, "On the buckling behavior of connected carbon nanotubes with parallel longitudinal axes," Applied Physics A, vol. 115, no. 4, pp. 13351344, 2014.

[3] S. K. Georgantzinos, G. I. Giannopoulos, and N. K. Anifantis, "An efficient numerical model for vibration analysis of singlewalled carbon nanotubes," Computational Mechanics, vol. 43, no. 6, pp. 731-741, 2009.

[4] W. Xia and L. Wang, "Vibration characteristics of fluidconveying carbon nanotubes with curved longitudinal shape," Computational Materials Science, vol. 49, no. 1, pp. 99-103, 2010.

[5] S. Arghavan and A. V. Singh, "On the vibrations of single-walled carbon nanotubes," Journal of Sound and Vibration, vol. 330, no. 13, pp. 3102-3122, 2011.

[6] A. Ghavamian and A. Öchsner, "Numerical modeling of eigenmodes and eigenfrequencies of single- and multi-walled carbon nanotubes under the influence of atomic defects," Computational Materials Science, vol. 72, pp. 42-48, 2013.

[7] F. N. Mayoof and M. A. Hawwa, "Chaotic behavior of a curved carbon nanotube under harmonic excitation," Chaos, Solitons and Fractals, vol. 42, no. 3, pp. 1860-1867, 2009.

[8] M. Farsadi, A. Öchsner, and M. Rahmandoust, "Numerical investigation of composite materials reinforced with waved carbon nanotubes," Journal of Composite Materials, vol. 47, no. 11, pp. 1425-1434, 2013.

[9] M. S. Dresselhaus, G. Dresselhaus, and R. Saito, "Physics of carbon nanotubes," Carbon, vol. 33, no. 7, pp. 883-891, 1995.

[10] C. Li and T.-W. Chou, "A structural mechanics approach for the analysis of carbon nanotubes," International Journal of Solids and Structures, vol. 40, no. 10, pp. 2487-2499, 2003.

[11] S. Melchor, F. J. Martin-Martinez, and J. A. Dobado, "CoNTub v2.0-algorithms for constructing C3-symmetric models of three-nanotube junctions," Journal of Chemical Information and Modeling, vol. 51, no. 6, pp. 1492-1505, 2011.

[12] Z. Kang, M. Li, and Q. Tang, "Buckling behavior of carbon nanotube-based intramolecular junctions under compression: molecular dynamics simulation and finite element analysis," Computational Materials Science, vol. 50, no. 1, pp. 253-259, 2010.

[13] T. Irvine, Application of the Newton-Raphson Method to Vibration Problems, Vibration Data Publications, Madison, Wis, USA, 1999.

[14] J. P. Lu, "Elastic properties of carbon nanotubes and nanoropes," Physical Review Letters, vol. 79, no. 7, pp. 1297-1300, 1997.

[15] C. W. S. To, "Bending and shear moduli of single-walled carbon nanotubes," Finite Elements in Analysis and Design, vol. 42, no. 5, pp. 404-413, 2006.

[16] A. L. Kalamkarov, A. V. Georgiades, S. K. Rokkam, V. P. Veedu, and M. N. Ghasemi-Nejhad, "Analytical and numerical techniques to predict carbon nanotubes properties," International Journal of Solids and Structures, vol. 43, no. 22-23, pp. 68326854, 2006.

[17] G. I. Giannopoulos, "Fullerenes as mass sensors: a numerical investigation," Physica E, vol. 56, pp. 36-42, 2014. 

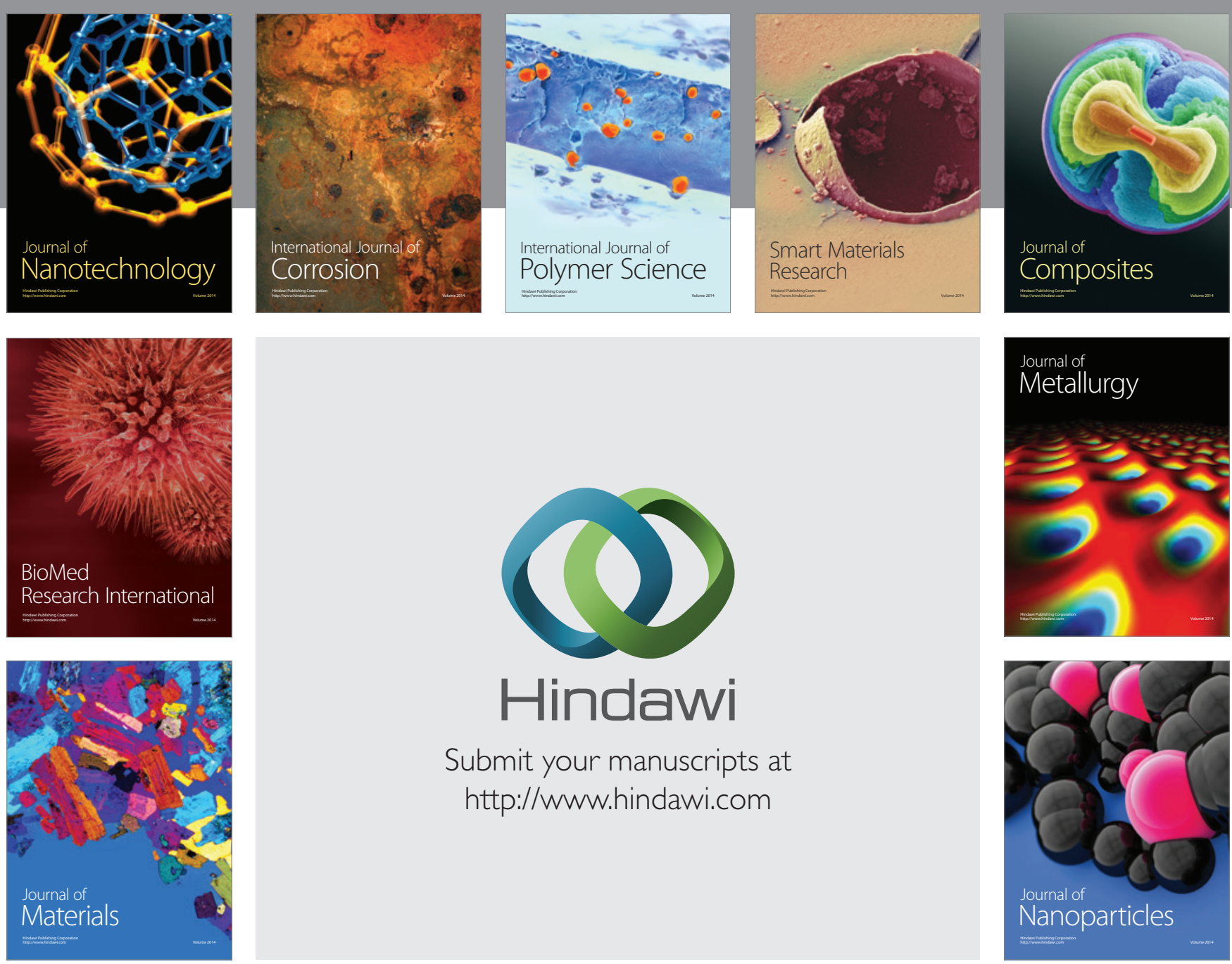

Submit your manuscripts at http://www.hindawi.com
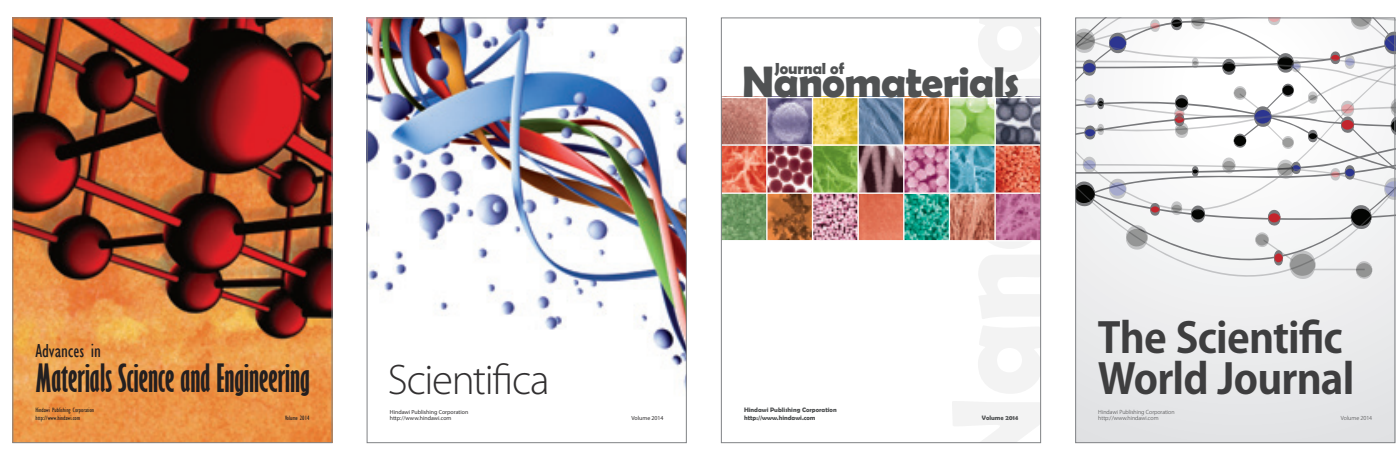

\section{The Scientific World Journal}
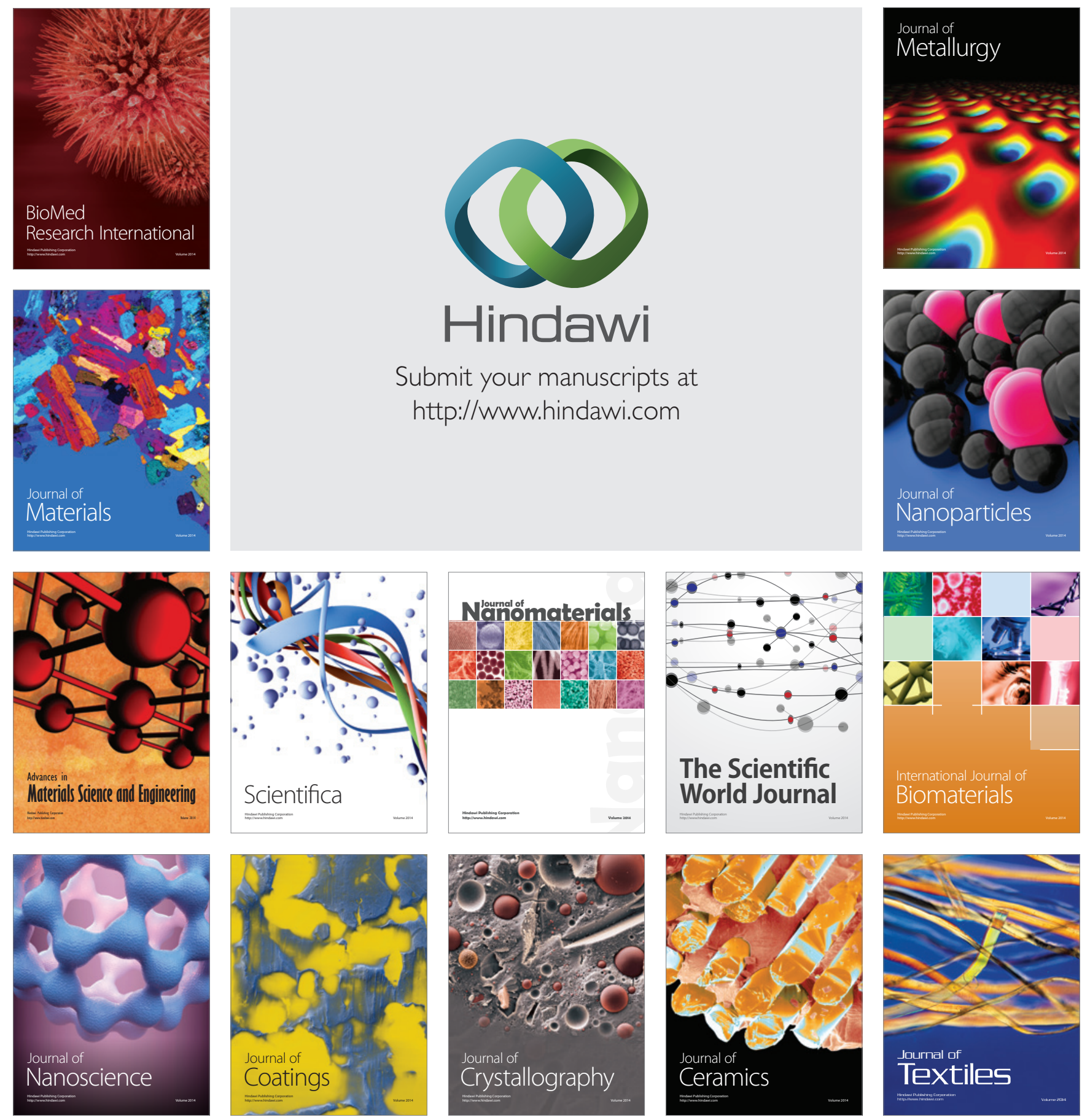